\title{
Erratum to: Inverse-Closed Algebras of Integral Operators on Locally Compact Groups
}

\author{
Ingrid Beltiţă and Daniel Beltiţă
}

\section{Erratum to: Ann Henri Poincaré DOI 10.1007/s00023-014-0320-x}

It was pointed out by Detlev Poguntke that the values of the map $\theta$ in the proof of Proposition 2.7 of original article may not be equivalence classes of Bochner integrable functions. Consequently the statements of Proposition 2.7, Corollary 2.8, Theorem 3.10, Theorem 5.2, and Corollary 5.3 of original article are not correct, and an extra hypothesis is needed in these statements in order to obtain the corresponding conclusions. Details in this connection can be found below.

One of the situations when the proof of Proposition 2.7 of original article applies is that of discrete groups, so that hypothesis will be added in the statement as follows.

Proposition 0.1. Let $G$ be a discrete group and $(\mathcal{A}, G, \alpha)$ be a $C^{*}$-dynamical system. Then there exists a $C^{*}$-dynamical system $(\overline{\mathcal{A}}, G, \bar{\alpha})$ with trivial action $\bar{\alpha}$ and an isometric $*$-homomorphism $\theta: \ell^{1}(G, \mathcal{A} ; \alpha) \rightarrow \ell^{1}(G, \overline{\mathcal{A}} ; \bar{\alpha})=\ell^{1}(G) \widehat{\otimes} \overline{\mathcal{A}}$.

Proof. The proof of Proposition 2.7 of original article applies in this case.

As a consequence, the hypothesis of Corollary 2.8 of original article should be strengthened by the assumption that the group under consideration is rigidly symmetric as a discrete group. This assumption is satisfied for the

The online version of the original article can be found under doi:10.1007/s00023-014-0320-x. 
nilpotent groups, which are the main examples that motivated the research recorded in the original article (see Example 3.11 of original article) and are also the main examples of non-compact rigidly symmetric groups known so far.

The corrected statement and proof of Corollary 2.8 of original article are provided below.

Corollary 0.2. Let $G$ be any locally compact group with its underlying discrete group denoted by $G_{d}$. Let $(\mathcal{A}, G, \alpha)$ be any $C^{*}$-dynamical system. If the group $G_{d}$ is rigidly symmetric, then the covariance algebra $L^{1}(G, \mathcal{A} ; \alpha)$ is a symmetric involutive Banach algebra.

Since the hypothesis that $G_{d}$ is rigidly symmetric was added in the above statement, one should also add that hypothesis in the statements of Theorem 3.10, Theorem 5.2, and Corollary 5.3 of original article, and in the proof of Theorem 3.10 of original article one should use the above Corollary 0.2 instead of Corollary 2.8 of original article.

For the proof of the above corollary we introduce some extra notation. To any $C^{*}$-dynamical system $(G, \mathcal{A}, \alpha)$ there corresponds the $C^{*}$-dynamical system $\left(G_{d}, \mathcal{A}, \alpha\right)$. If $\mathfrak{B}:=L^{1}(G, \mathcal{A} ; \alpha)$ and $\mathfrak{B}_{d}:=\ell^{1}\left(G_{d}, \mathcal{A} ; \alpha\right)$ are their covariance algebras, then every simple $\mathfrak{B}$-module $E$ can be made into a nondegenerate contractive Banach $\mathfrak{B}$-module (see [5, page 191]).

Definition 0.3. Every nondegenerate contractive Banach $\mathfrak{B}$-module $E$ is a nondegenerate contractive Banach $\mathcal{M}(\mathfrak{B})$-module in a canonical way by [2, Th. 4.5(1)], where $\mathcal{M}(\mathfrak{B})$ is the multiplier algebra of $\mathfrak{B}$. Then for $\mathfrak{B}=$ $L^{1}(G, \mathcal{A} ; \alpha)$ one obtains by [3, Prop. 2.1] a covariant representation of $(G, \mathcal{A}, \alpha)$ on $E$, hence a covariant representation of $\left(G_{d}, \mathcal{A}, \alpha\right)$ on $E$, and eventually a structure of $\mathfrak{B}_{d}$-module on $E$, called the discretization of the nondegenerate contractive Banach $\mathfrak{B}$-module $E$.

Lemma 0.4. Let $\mathcal{X}$ and $\mathcal{Y}$ be any Banach spaces. If $T: \mathcal{X} \rightarrow \mathcal{Y}$ is a bounded linear operator satisfying the condition

$$
(\forall y \in \mathcal{Y})(\forall \epsilon>0)(\exists x \in \mathcal{X}) \quad \max \{\|x\|-\|y\|,\|T x-y\|\}<\epsilon
$$

then $T$ is surjective.

Proof. See the final paragraph of the proof of [5, Th. 2].

Lemma 0.5. For every simple $\mathfrak{B}$-module $E$, its discretization is a simple $\mathfrak{B}_{d^{-}}$ module.

Proof. Denote the $\mathfrak{B}$-module structure of $E$ by $\mathcal{B} \times E \rightarrow E,(f, y) \mapsto \rho(f) y$ and the $\mathfrak{B}_{d}$-module structure of $E$ by $\mathcal{B} \times E \rightarrow E,(\varphi, y) \mapsto \rho_{d}(\varphi) y$. To prove that the $\mathfrak{B}_{d}$-module $E$ is simple, one checks that for arbitrary $y_{0} \in E$ the operator $\mathfrak{B}_{d} \rightarrow E, \varphi \mapsto \rho_{d}(\varphi) y_{0}$ is surjective. To this end we use Lemma 0.4. The hypothesis of that lemma is satisfied with the norm on $E$ that comes from the fact that the simple $\mathfrak{B}$-module $E$ can be made into a nondegenerate contractive Banach $\mathfrak{B}$-module as noted above. The method of proof of $[5$, Th. 2] carries over to the present setting. 
Proof of Corollary 0.2. By using Proposition 2.7 of original article for the discrete group $G_{d}$, it follows that the covariance algebra $\ell^{1}\left(G_{d}, \mathcal{A} ; \alpha\right)$ is isometrically $*$-isomorphic to a closed involutive subalgebra of $\ell^{1}(G, \overline{\mathcal{A}} ; \bar{\alpha}) \simeq \ell^{1}(G) \widehat{\otimes} \overline{\mathcal{A}}$ for a suitable $C^{*}$-algebra $\overline{\mathcal{A}}$. On the other hand, the involutive Banach algebra $\ell^{1}\left(G_{d}\right) \widehat{\otimes} \overline{\mathcal{A}}$ is symmetric since the discrete group $G_{d}$ is rigidly symmetric. Now, since any closed involutive subalgebra of a symmetric Banach algebra is in turn symmetric (see for instance [1, Prop. 7.10]), it follows that $\ell^{1}\left(G_{d}, \mathcal{A} ; \alpha\right)$ is a symmetric Banach algebra.

To prove that also $L^{1}(G, \mathcal{A} ; \alpha)$ is symmetric, let $E$ be any simple $L^{1}(G, \mathcal{A} ; \alpha)$-module. The discretization of $E$ is a simple $\ell^{1}\left(G_{d}, \mathcal{A} ; \alpha\right)$-module by Lemma 0.5 . We proved above that $\ell^{1}\left(G_{d}, \mathcal{A} ; \alpha\right)$ is a symmetric Banach algebra, so by $[4,(1)]$ there exists a continuous positive sesquilinear form $(\cdot \mid \cdot)$ on $E$ with $\left(\rho_{d}(\phi) x \mid y\right)=\left(x \mid \rho_{d}\left(\phi^{*}\right) y\right)$ for all $x, y \in E$ and $\phi \in \ell^{1}\left(G_{d}, \mathcal{A} ; \alpha\right)$. By using the method of proof of $[5$, Cor. 6] one can then prove that

$$
(\rho(f) x \mid y)=\left(x \mid \rho\left(f^{*}\right) y\right) \text { for all } x, y \in E \text { and } f \in L^{1}(G, \mathcal{A} ; \alpha) .
$$

Thus $L^{1}(G, \mathcal{A} ; \alpha)$ is a symmetric algebra by $[4,(1)]$, and this concludes the proof.

\section{References}

[1] Biller, H.: Continuous inverse algebras with involution. Forum Math. 22(6), 10331059 (2010)

[2] Dirksen, S., de Jeu, M., Wortel, M.: Extending representations of normed algebras in Banach spaces. In: de Jeu M., Silvestrov, S., Skau, C., Tomiyama, J. (eds.) Operator Structures and Dynamical Systems Contemporary Mathematics, vol. 503, pp. 53-72. American Mathematical Society, Providence (2009)

[3] Lau, A.T., Paterson, A.L.T.: Amenability for twisted covariance algebras and group $C^{*}$-algebras. J. Funct. Anal. 100(1), 59-86 (1991)

[4] Leptin, H.: Symmetrie in Banachschen Algebren. Arch. Math. (Basel) 27(4), 394400 (1976)

[5] Poguntke, D.: Rigidly symmetric $L^{1}$-group algebras. Sem. Sophus Lie 2(2), 189197 (1992)

Ingrid Beltiţă and Daniel Beltiţă

Institute of Mathematics "Simion Stoilow" of the Romanian Academy

P.O. Box 1-764, Bucharest, Romania

e-mail: ingrid.beltita@gmail.com; Ingrid.Beltita@imar.ro;

beltita@gmail.com; Daniel.Beltita@imar.ro 